\title{
Content Authoring and Cloud System Implementation for Smart Learning
}

\author{
Hyun-Seo Hwang1, Jin-Tae Park1, Kyeong-Taek Seo1, Jun-Soo Yun1, Il-Young Moon1, \\ Oh-Young Kwon', Byeong-Jun Kim² \\ ${ }^{1}$ School of Computer Science and Engineering, University of Technology and Education, Cheon-an, Korea \\ ${ }^{2}$ ANCLE Inc, Cheon-an, Korea \\ Email: smilebear1@koreatech.ac.kr
}

Received April 2015

\begin{abstract}
In this paper, we implement a content authoring and cloud system for cloud-based smart cloud learning. With the advent of the smartphone and mobile devices such as tablets, the educational paradigm is also changing. Was using the computer to aid in the learning e-learning started in ICT education are evolving Over the variety, in recent years, combines smart learning and social learning, cloud-based smart devices in e-learning the concept of Smart Cloud Smart Education Services Learning was gives rise to the term. Smart Cloud and free running is always the knowledge content uploaded by anyone, anywhere, can also be shared with other users. A terminal and location, without limitations on time, are continuously available to the environment of a highquality knowledge content was coming through a variety of smart media, open educational content platform is built over the level of the possible conditions than e-learning. In this paper, we develop applications and Web sites that can provide authored content for smart cloud learning. In addition, we have built the cloud for content management and the website that can share content with other users. In the future, should wish to study a way that can provide customized services over the learner analysis based on big data technology.
\end{abstract}

\section{Keywords}

Smart Cloud Learning, Smart Learning, E-Learning, Big Data, Mobile Device

\section{Introduction}

According to the increasing market for mobile devices, the education paradigm is changing [1]. Recently, springing to the term smart learning and social learning, smart cloud learning. Smart Cloud learning is a smart educational service in e-learning that combines cloud-based smart devices [2]. Cloud has been frequently mentioned recently. In smart environments, coupled with the Big Data concept, smart cloud learning has been recognized as having an absolute value. Cloud services are available on the Internet whenever you need to save your data or software in the data center rather than to individual devices [3]. Movies, photos, music, media files, and other content can be stored on a server that users can download from any device, including smartphones and 
smart TV. The computing resources that exist in different physical locations, such as the hardware and software computing resources in the form of immaterial to the computing services as they need to use the technology provided by a virtualization technology [4] [5]. Smart Cloud learning is a smartphone or tablet PC, such as smart devices and cloud services environments, learner-centric, self-directed, and the interaction, intelligent, nonformal learning, e-learning a new technology that can enhance the realism of new fusion educational services [6]. Figure 1 shows a smart learning configuration diagram of a cloud computing-based.

In the smart cloud learning system, content providers can upload content anytime, anywhere, regardless of where the user is located. Smartphone users also can take advantage of the content, regardless of the place where the content was loaded, such as a tablet PC. Leads to new knowledge and technologies utilize an independent, intelligent changes of learner behavior through education, utilizing smart infrastructure build mutual learning and collective intelligence enables learning styles such as traditional e-learning is never acceptable [7]. In this paper, we developed a smart cloud learning content authoring service that can be provided directly to the Apple application servers and web sites; we developed a web site that allows you to manage your content. In addition, by building a cloud, we can store a variety of content and implement it for users [8].

\section{Related Research}

\subsection{Smart Learning}

Smart learning is the learning content and solutions that use smart phones and mobile devices such as the tablet PC. Internet access is a training method that takes advantage of smart devices, as well as location-based services, and a variety of techniques, such as augmented reality. Smart learning is simply not meant to be e-learning in another form using the mobile device or the smart devices [9]. Smart learning considers a variety of learning styles and abilities of learners and learners' thinking, communication, and problem solving skills, and improves development, such as with cooperative learning and individual learning, by creating opportunities for people to make the device more enjoyable than learning to learn and an advanced ICT infrastructure of intelligent personalized learning for effective learner-centered content [10]. Citing benefit0073 of smart learning is learning seep into the life out of the formal form can quickly obtain the required information or knowledge. Smartphone, while the prevalence of mobile devices such as tablet PC popularity mobility, personalization, mobile devices are having such a characteristic can learn timeliness of the content that can be implemented by using the most appropriate device.

\subsection{Content Authoring Tool}

The user is required to upload content using a learning content authoring tool. Users should be able to use a content authoring tool allowing them to easily and conveniently do this task. At tekvile.com, we launched a service for PC and mobile devices simultaneously as the work of only one possible HTML5-based smart learning content authoring tool, WebCAT. WebCAT enables writing smart learning content easily, quickly, and economically. The WebCAT supports the next-generation standard for HTML5, which has the advantage of

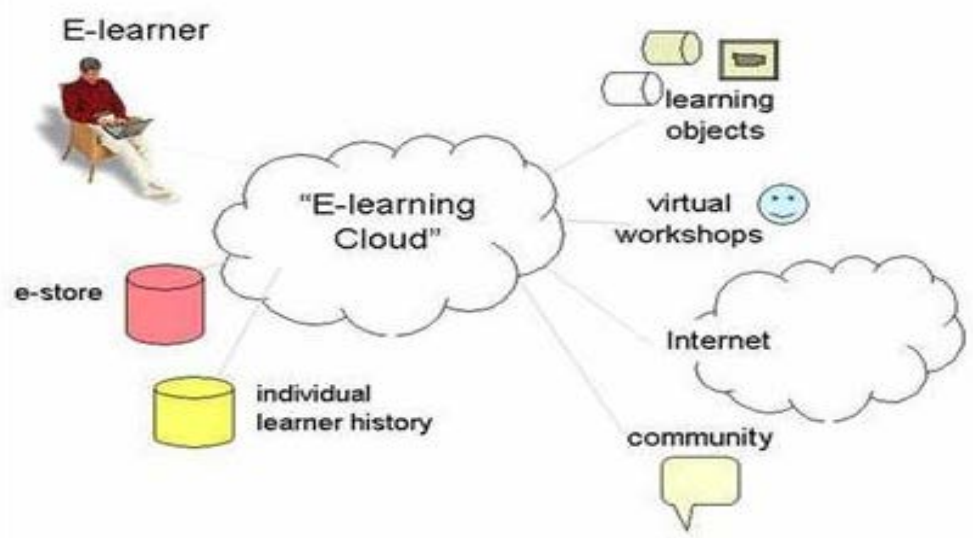

Figure 1. Smart learning configuration diagram of cloud computing base. 
corresponding to a variety of devices, such as a PC, as well as using the PC and the mobile device as a source of a Web standard technique that can be used for interactive content in the mobile device. Existing e-learning content created with Flash is supported as author content for mobile devices because it does not work well on obsolete mobile devices, for MP4 or HTML5 technology. However, HTML5 was an e-learning content production company that has been costly and time-consuming when converting existing Flash content into a movie. WebCAT can be used for both PC and mobile device content to HTML5 authoring tools for smart learning. Further, the cost can be easily and quickly authoring the content, the learning content development and promoted the user's convenience with the modularity of the various functions required. WebCAT addition to a variety of content authoring tool has been released, the next release of e-learning content created with Flash will be replaced by the most recent HTML5.

\section{System Implementation}

\subsection{Smart Learning}

Users must have can be managed, such as video and music taken through the Web site of the PC environment. Web site not only to build the PC environment, and configure the UI to allow easy use in a mobile environment. As a function of this site, large, video control, audio control, settlement work, and content publishing, are configured in such content viewing. As Figure 2 shows, is a form of work web site content in a PC environment, Figure 3 shows a UI design in mobile environments. In the PC and mobile environments, by changing the position to fit the same content to the resolution, ease of use, and to provide an environment.

- System Design

Content Management Website, edx-platform the developed base. On the basis of the lms and cms resolution $760 \mathrm{px}$ after creating the web site, if the size of the browser is smaller than the reference size, by changing the layout, mainly the description and indexes river beside, place, contents, you can get down to the bottom, each component has, you configure the layout to use the entire screen

- CMS and Cloud Collaboration

Log put the account information to the cloud interface with post format when you login to Cms. Together and allowed to login sure that you save the cloud session, so as to use when calling the url of cloud. The cloud.url page is configured to have no sense of incongruity can be directly borrowed UI upload page of an existing file. In the Cloud url page, and then split the only name of the video url and video and read the source of the cloud of interface in ajax, to configure the table on the results of the value. When users upload to the cloud from cms, the upload will be sent directly to the cloud interface. In cms, after the data ascends to the cloud, the updated file is to be displayed on the added screen page. The user, after selecting the video component in the unit of making a lecture by copying the individual url and pasting the address. Because I have configured as lectures that have been created in the user look.

- Learning Object and Cloud Upload

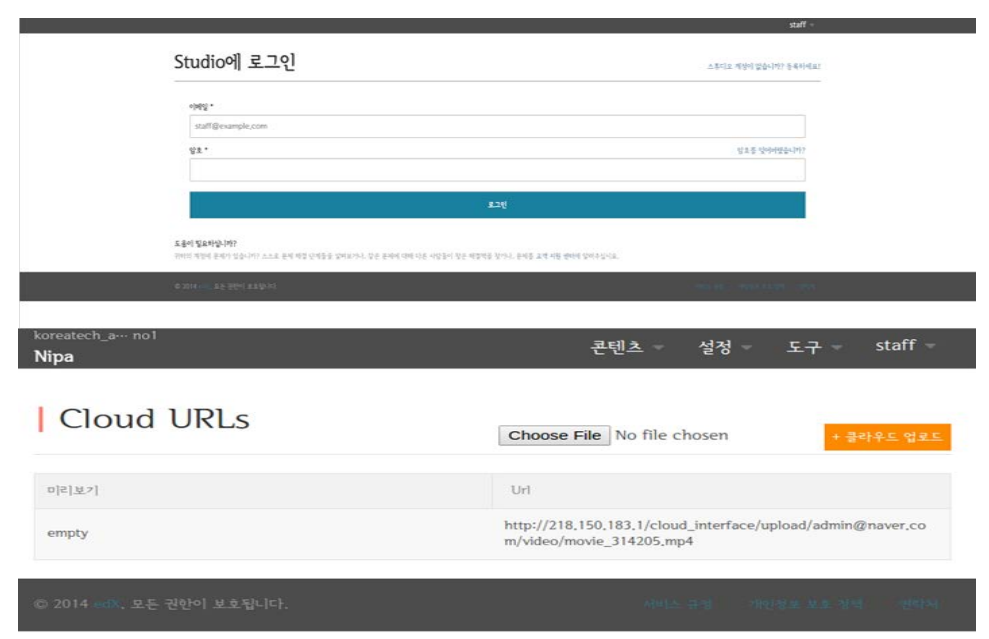

Figure 2. Content management web site. 


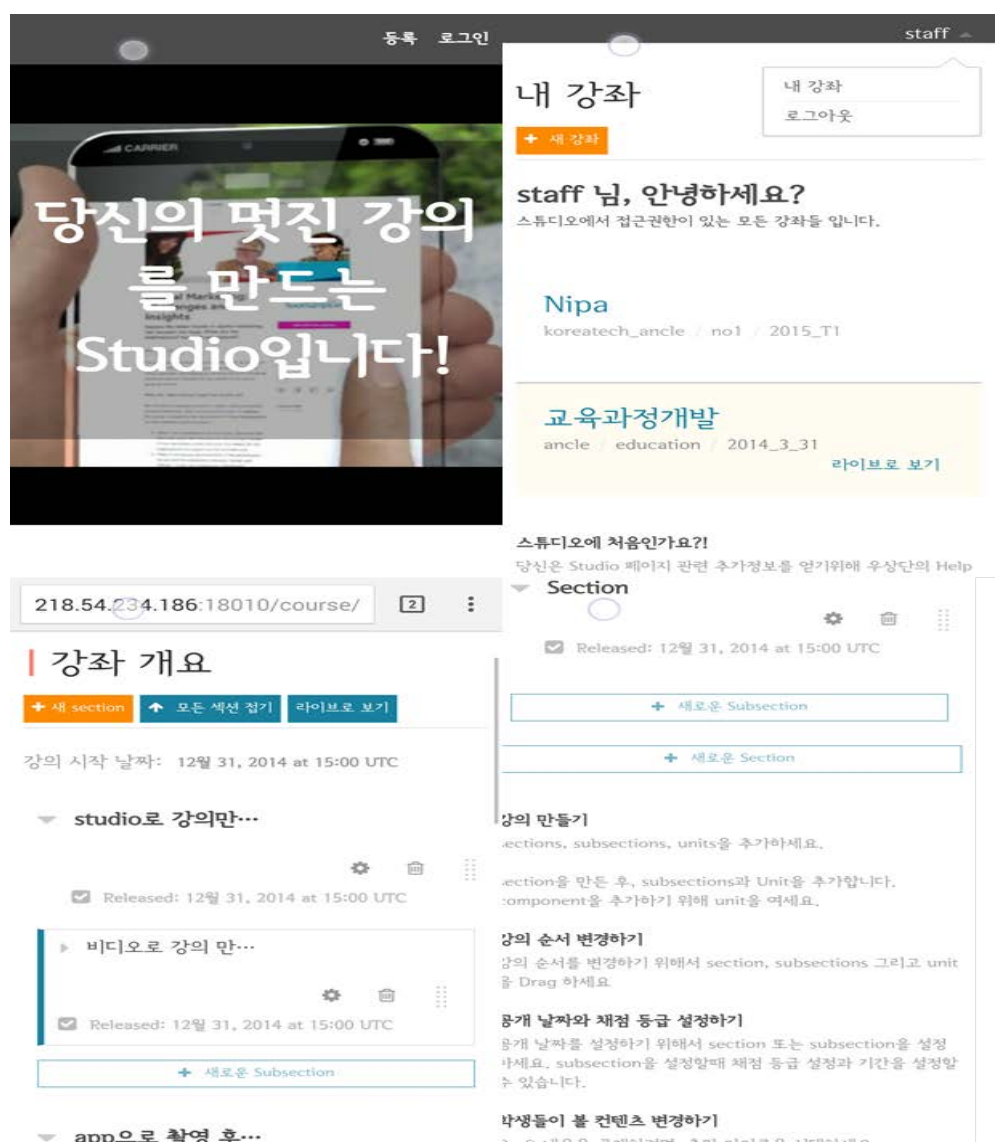

Figure 3. Content management mobile application.

The learning_object when you export the lecture has been downloaded to the PC in tar format, which is then directly uploaded to the cloud; it is stored in a cloud of learning objects folder. After this, the import function lecture file is designed to load the saved course. The learning object is composed of a folder, as shown in Figure 4, starting from course.xml. There is a guided lecture and static materials such as video, images used in the river, and policies so that student scoring is carried out in a lecture, video, vertical, sequential, html, and the video contents of the lecture; a reference value of the sub-data is required.

\subsection{Content Authoring Application}

A general device that is capable of creating the simplest content for the user is a smart phone. By using smartphones, movies, and photos, as music is used, it is possible to use the content. By using the smart phone, movies, photos, etc. by using the music, it is possible to easily use the content. A content authoring application was developed to fit the Android operating system, to develop 4.4.2 of the Android with a minimum version. The function of the application, and in my lecture you can see content that I wrote, lecture shooting that can shoot the content, lectures can edit the content editing, lectures can upload your wrote the content to the cloud server, upload and configured. Figure 5 shows a state of the content authoring application.

- My Lecture

The user must be able to check by using an application developed for self-written content. Therefore, creates a menu that my lecture was to be able to check the pictures and videos using stored on his smartphone Gallery feature of Android. Media player, use the default media player that is built into the smartphone. Default reason for using the media player uses a media player that is built to improve the compatibility of multiple users of Smartphone.

- Make Lecture

User to configure the menu called simply lectures to be able to create content. Users can take photos and vid- 


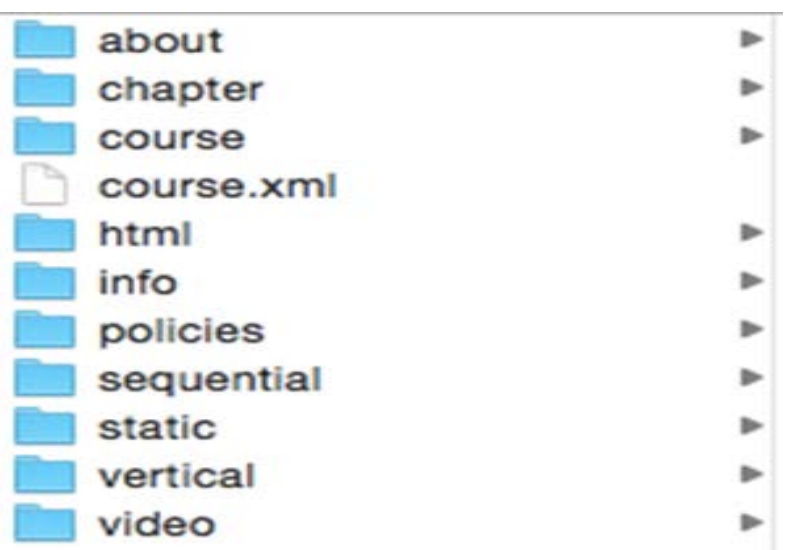

Figure 4. Structure of the learning object.

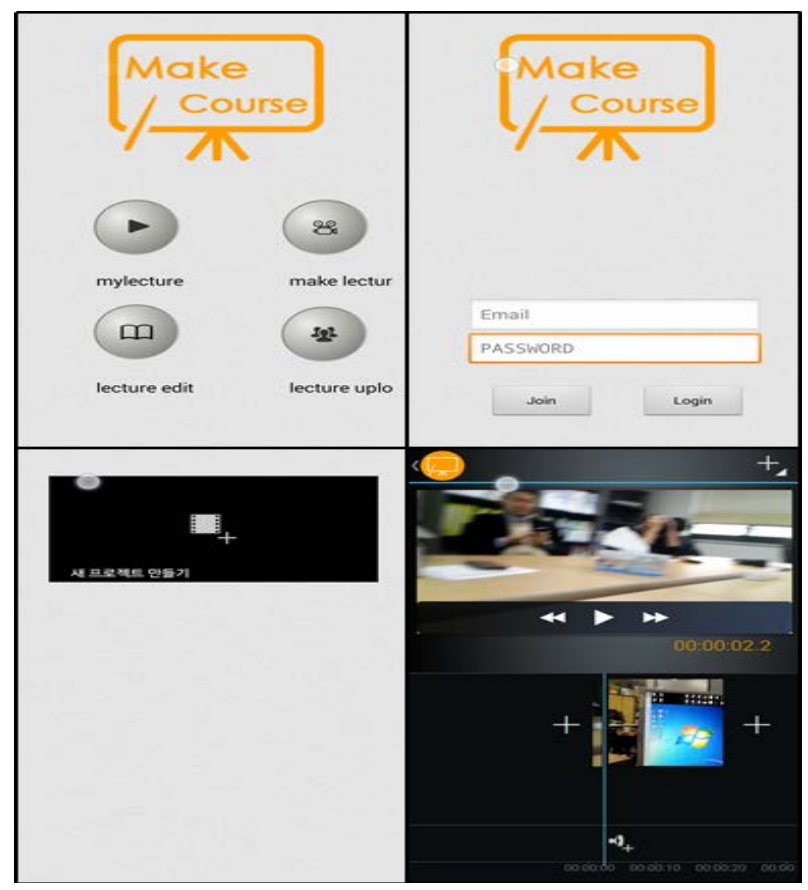

Figure 5. Content authoring application.

eo using a camera that is built as a smartphone. The captured image is to be stored in the smart phone. Location of the video, specifically the make_course smartphone sdcard, to ensure there is no confusion with the existing video file.

- Edit Lecture

Before you can upload the content to the cloud server, users can edit the lecture in the direction you want. Editing lectures first is configured to create a project, which can be edited by project basis and may be uploaded. Within the project, to produce a video in a way that is summarized by adding a variety of video stored on the smartphone. When it is converted from the current image to the next image, it is possible to add a transition effect that was created previously. Moreover, by inserting the background music, the user's gradation can be a video production atmosphere that enables sepia tones, and application of image filter effects, such as negatives. In addition, the function required for authoring content is the subtitle function. In this application, so as be able to subtitle when a user likes to center the lower end of the video. Video completed editing, via the encoding process is stored in the internal user of the smartphone. Location of the video that has been saved using the editing is in a specified folder. 
- Upload Lecture

By editing the lectures, authored content is uploaded to the cloud server through a lecture upload. Content httpPost, by using the httpClient method, is uploaded to the specified php file. Prior to the uploading of the content to implement the login function based on the information of the cloud interface that is stored in the cloud server. Then, the content in the application folder is created in the cloud server with a user email name. In the generated folder, video, image, are three of the folder called learning_object is generated. Each folder will be stored by classifying the content uploaded by users. In addition, the upload.php received the upload request, and stored the user's email database specified by a request to post information, and to create a file name and a storage path of the file.

\subsection{Building a Cloud Service}

Cloud computing provides a "Virtualized Resources" service. Using the Internet, users can borrow and use resources such as software, storage server, Network as much as they need. Users can gain real-time expandability through load balancing services and they only pay for the amount they have used In this paper, using the Open Stack, one open source to build a Cloud platform and want to build a Cloud environment for the service.

\subsubsection{Cloud System}

Uses three nodes to establish a cloud for service. in the controller node, Database is MySQL and message services use RabbitMQ. Services are installed such as Keystone for authentication, Glance for image management, Compute service in Nova, Horizon Dashboard service is to give convenience to the user. Including a network service from Nova without using Neutron it was configured to use the compute node Nova-network without installing a separate network service. Compute nodes KMV hypervisor is installed with nova-network that support network services for instance. Controller node requires one interface for monitoring and management. Compute nodes, one interface for the controller nodes and connections and One interface for communication with the external network So with two interfaces were built. Installation of OpenStack can be installed through the services database to create a separate service, additional credentials for that service in Keystone, service installation, configuration, installation, service made to restart in order to provide OpenStack documents. Figure 6 shows a block diagram of an openstack.

\section{Conclusion}

With the advent of mobile devices, the paradigm of education has changed; there has been a growing interest in smart learning. To the existing e-learning following, smart learning, and social learning, smart education service was developed that connects smart devices such as smart cloud learning on e-learning. Recently, online public

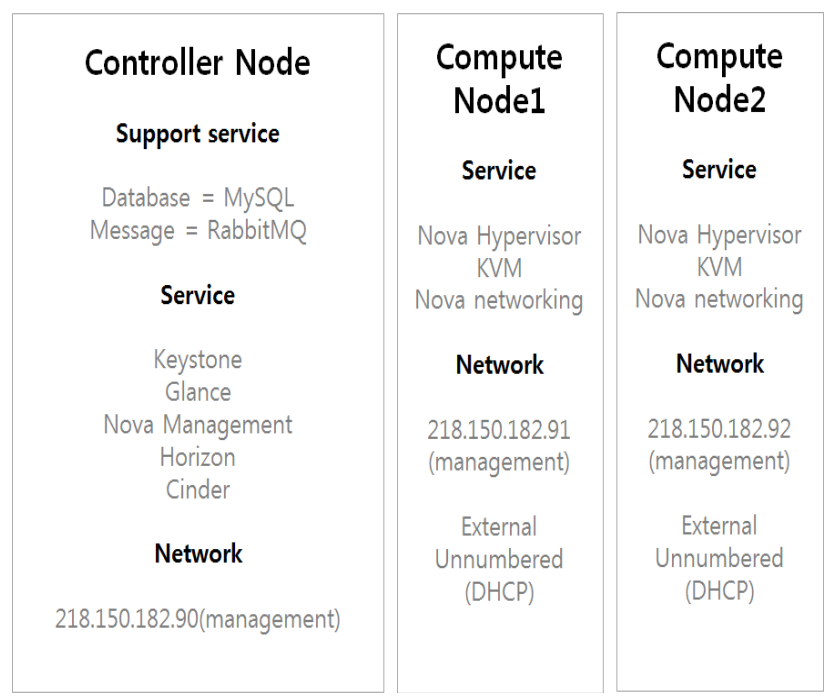

Figure 6. OpenStack configuration diagram. 
lectures that globally Massive Open Online Course (MOOC) has attracted attention. MOOC is a large-scale online public lecture through which you can receive the education of content through anyone without restrictions online. Lectures provided by the MOOC include course information, courses, quizzes, essays, and discussion, are constituted by a question bulletin board. In addition, the MOOC is enclosing three important meanings. First, humans deserve a good education; second, these innovative solutions reduce training costs. The third is the place of formation of the intellectual network. Everyone regardless of where as online course systems that MOOC is provided which is implemented in this paper, it has been developed to be able to provide online courses over the Internet. In the future, through our proposed system, we expect to democratize education beyond Korea.

\section{Acknowledgements}

This treatise is conducted with funding (H0502-14-2007) from the ICT/SW creative research process business of the National IT Industry Promotion Agency (NIPA) of the Ministry of Science, ICT and Future Planning (MSIP).

\section{References}

[1] Lee, J.G. and Lee, J.W. (2010) Interface for in-Situ Authoring of Augmented Reality Contents on a Mobile Device Environment. Korea Contents Society, 10, 1-9.

[2] Lee, J.H., Song, J.H., Oh, J.M., Choi, Y.J. and Moon, N.M. (2010) The Study on Improvement of Moving Picture Content Authoring Tool for Usability Enhancements. Journal of the HCI Society of Korea, 10, 242-245.

[3] Son, S.H. and Kang, D.J. (2014) Development of a Testing Tool to Validate Integrity of a Constructed Cloud System based on OpenStack. KISE Transactions on Computing Practices, 20, 658-663. http://dx.doi.org/10.5626/KTCP.2014.20.12.658

[4] Lee, K.C., Lee, S.Y., Young, H.D. and Park, C.Y. (2014) Development of Service Verification Methodology Based on Cloud Computing Interoperability. Journal of Security Engineering, 11, 299-312. http://dx.doi.org/10.14257/jse.2014.08.03

[5] Ra, J.H., Han, S.H., Sung, B.Y. and Kim, Y.K. (2012) Open Source Cloud Platforms: OpenStack and CloudStack. Communications of the Korean Institute of Information Scientists and Engineers, 39, 259-261.

[6] Lee, H.J. and Choi, H.S. (2008) Development of LMS Templates to Motivate Professors' Use of an e-Learning System. Korean Society for Educational Technology, 24, 153-180.

[7] Jeong, H.Y., Kim, E.W. and Hong, B.H. (2008) A Scheduling of the Multimedia Contents Processing in LMS for E-Learning System. Journal of the Institute of Electronics Engineers of Korea, 45, 50-57.

[8] Seo, K.T., Hwang, H.S., Moon, I.Y., Kwon, O.Y. and Kim, B.J. (2014) Comparative Analysis of LXC Docker and KVM Performance. International Journal of Applied Engineering Research, 9, 29891-29900.

[9] Lee, S.T. and Yu, H.B. (2013) Analysis of K-Defense Cloud Computing Service Availability Considering of Cloud Computing Traffic Growth. Convergence Security Journal, 13, 93-100.

[10] Park, J.T., Yun, J.S., Kwon, O.Y., Moon, I.Y. and Kim, B.J. (2014) Development of a Smart-Learning Platform and Content Authoring Tools that Uses a Cloud and Web. International Journal of Applied Engineering Research, 9, 29869-29882. 